\title{
Decay of trapped-particle asymmetry modes in non-neutral plasmas in a Malmberg-Penning trap
}

Grant W. Mason

masong@utah-inter.net

Follow this and additional works at: https://scholarsarchive.byu.edu/facpub

Part of the Astrophysics and Astronomy Commons, and the Physics Commons

\section{Original Publication Citation}

Mason, Grant W. "Decay of trapped-particle asymmetry modes in non-neutral plasmas in a Malmberg-Penning trap." Physics of Plasmas 1 (23): 1231-1238.

\section{BYU ScholarsArchive Citation}

Mason, Grant W., "Decay of trapped-particle asymmetry modes in non-neutral plasmas in a MalmbergPenning trap" (2003). Faculty Publications. 496.

https://scholarsarchive.byu.edu/facpub/496 


\title{
Decay of trapped-particle asymmetry modes in non-neutral plasmas in a Malmberg-Penning trap
}

\author{
Grant W. Mason ${ }^{\text {a) }}$ \\ Department of Physics and Astronomy, Brigham Young University, Provo, Utah 84602
}

(Received 4 December 2002; accepted 19 February 2003)

\begin{abstract}
The mechanism for the strong damping of diocotron-like azimuthal trapped-particle asymmetry modes in a Malmberg-Penning trap is investigated with a detailed three-dimensional particle-in-cell computer simulation. The $m=1, k_{z} \neq 0$ modes are created by a voltage squeeze from a mid-detector ring followed by a displacement of trapped particles in opposite directions on either side of the ring. The voltage squeeze creates a population of particles confined to half the trap length (trapped) and a population of particles that move longitudinally along the full length of the cylinder (untrapped). The damping of the modes is found to be the result of radial transport relative to the $m=1$ mode (charge) center caused by transitions of particles from untrapped-to-trapped states induced by diffusion of the particles in velocity space. The transport is the immediate consequence of a difference in dynamical orbits for trapped and untrapped particles. The random walk in velocity space results in particles repeatedly changing state from trapped to untrapped and back. The dependence of the mode frequency and the exponential decay constant are explored as a function of squeeze voltage, magnetic field, and temperature in order to establish scaling behavior. (C) 2003 American Institute of Physics. [DOI: 10.1063/1.1566959]
\end{abstract}

\section{INTRODUCTION}

Non-neutral plasmas, typically ions or electrons, can be confined for long periods of time in a cylindrical MalmbergPenning trap similar to that shown in Fig. 1. A stiff axial magnetic field confines the particles radially and charged rings at the ends of the otherwise grounded cylinder provide electrostatic longitudinal confinement. Diocotron modes are azimuthal drift waves in the cylindrical plasma that vary spatially as $\exp (\operatorname{im} \theta)$. The theory of diocotron modes in nonneutral plasmas has its origins in seminal papers by Levy, ${ }^{1}$ Briggs, Daugherty, and Levy, ${ }^{2}$ and the comprehensive treatment of non-neutral plasmas by Davidson. ${ }^{3}$ Experimental work at the University of California San Diego for more than a decade has also contributed particularly to the foundation of understanding of these modes. ${ }^{4}$

Of particular interest in this paper is the $m=1$ mode in which the plasma is offset from the symmetry axis of the trap. In this configuration the plasma does an $\mathbf{E} \times \mathbf{B}$ drift around its own symmetry axis in the electric field arising from its own space charge. The rotation has a radial dependence referred to as the plasma's "rotation frequency profile." The offset plasma as a whole also revolves about the symmetry axis of the trap. The latter motion can itself be thought of as an $\mathbf{E} \times \mathbf{B}$ drift in the electric field generated by induced charge in the grounded wall of the cylinder. The latter motion is the so-called $m=1$ "wall mode" and has as its revolution frequency (for infinite length) the value of the rotation frequency profile taken at the radius of the cylinder. For a symmetric plasma with longitudinal wave number $k_{z}$ $=0$, the mode is stable.

${ }^{\text {a)} E l e c t r o n i c ~ m a i l: ~ m a s o n g @ e i n s t e i n . b y u . e d u ~}$
Here we consider a modification of the diocotron mode when an applied "squeeze voltage" is applied to an additional ring installed at the longitudinal center of the trap and the plasma is offset from the symmetry axis in opposite directions on each side of the center ring such that the parity of $k_{z} \neq 0$ is odd. The squeeze voltage creates an energy barrier at the longitudinal median plane that gives rise to a population of trapped particles on either side of the divide as well as a population of untrapped particles with sufficient energy to traverse the entire length of the trap. The result is a new mode revolving at a frequency different from the wall value of the rotation frequency profile. These "trapped-particle asymmetry modes" have been experimentally observed and reported by Kabantsev et al. ${ }^{5,6}$ In particular, the modes were strongly damped by a mechanism that the authors originally described as "not yet understood." ${ }^{\prime}$ Spatial Landau damping and diffusive velocity mixing of trapped and untrapped particles were suggested as possible mechanisms. The dependence of the decay constant on magnetic field has been particularly problematic. The earliest published data for the modes ${ }^{5}$ showed the decay constants increasing with magnetic field strength. Later the decay of the modes was reported to have no dependence on the strength of the magnetic field over a range $0.5 \mathrm{kG} \leqslant B \leqslant 10.0 \mathrm{kG}^{6}{ }^{6}$ More recently these two earlier claims have been retracted, ${ }^{7,8}$ noting that the magnetic field dependence was missed earlier due to concurrent plasma temperature changes. The most recent data are divided into two regimes, one at lower magnetic fields varying roughly as $B^{-1}$ eventually giving way to a less steep dependence $\left(B^{-0.5}\right)$ at higher magnetic fields. ${ }^{8}$ Hilsabeck and O'Neil have ascribed the damping mechanism to velocity scattering of marginally trapped particles. They have devel- 


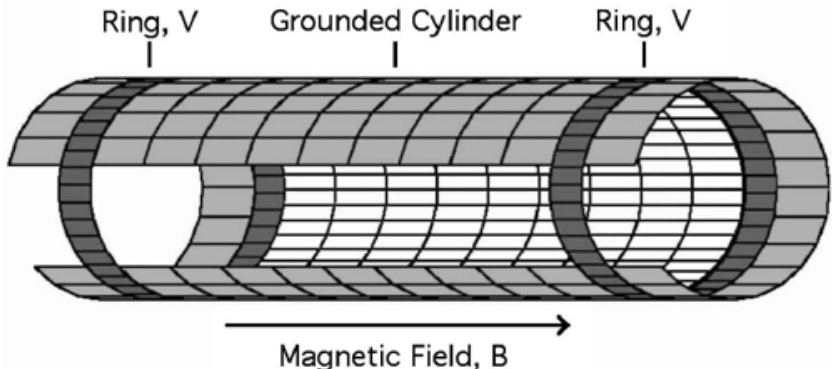

FIG. 1. A Malmberg-Penning trap. The axial magnetic field $B$ confines charged particles radially and voltages $V$ on the rings confine the non-neutral plasma longitudinally in the cavity space between the rings.

oped a theory based on this mechanism for which they report good agreement with measurements. ${ }^{9}$

In this paper we report the results of detailed threedimensional computer simulations of the trapped-particle asymmetry modes. ${ }^{10}$ The decay mechanism is demonstrated to be a consequence of the orbital dynamics of particles moving back and forth between the trapped and untrapped populations. The interchange between the two populations is a result of slow modulations (diffusion) of the longitudinal velocities of the particles. We also demonstrate that the decay of the mode does, in fact, depend on the strength of the magnetic field (varying in the simulations as $B^{-0.5}$ ). We also demonstrate the dependence of the decay constant on temperature in the simulations for a narrow range of temperatures.

In Sec. II we describe the model and computer simulations. In Sec. III we consider the results of the simulations and in Sec. IV we summarize our conclusions.

\section{SIMULATIONS}

We take a numerical approach by doing particle-in-cell (PIC) simulations using a code described previously. ${ }^{11}$ The method has the advantage of incorporating realistic boundary and end conditions in detail as well as the full threedimensional nature of the trapped-particle asymmetry modes. We perform numerical experiments in which, if necessary, we can follow the orbits of individual particles with the intent of helping to understand the mechanism driving the decay of the modes. The disadvantage of the PIC approach is that the code uses strictly drift motion in the plane perpendicular to the length of the cylinder while using Newton's second law in the longitudinal direction. The result is that "collisions" of particles in the model only affect longitudinal motion. Moreover, the simulation particles followed in the PIC simulations each represent some larger number of real particles and hence carry a larger charge and larger mass than real particles. The consequence of this fact is that fluctuations in longitudinal velocity are exaggerated and this exaggeration must be removed before actual comparison with experiments is possible. However, the PIC simulations should be adequate for exploring the mechanism for transport and mode decay and for determining scaling behavior if the phenomenon is essentially a consequence of longitudinal collisions.

To begin the simulations azimuthally symmetric equilibria are computed separately using a two-dimensional $(r$ $-z$ ) nonlinear Poisson solver. ${ }^{12}$ The two-dimensional density array is passed from the equilibrium code to the simulation code and interpolated onto a three-dimensional Cartesian grid. The three-dimensional density is then represented by particles-in-cells (PIC).

In the present work, the plasma is typically represented by about $1.5 \times 10^{6}$ computational "particles" that, in turn, each represent about 450 plasma electrons. The computation is done in three-dimensional Cartesian geometry into which is embedded the confining cylinder. The grid used was $N_{x}$ $\times N_{y} \times N_{z}=65 \times 65 \times 129$. Short-legged differential operators for the Laplacian operator are used at the cylindrical boundary so that the cylindrical shape is treated realistically. Likewise, boundary conditions are implemented realistically, with a grounded cylinder sandwiched between confinement rings at each end held at sufficient potential to confine the plasma. (See Fig. 1.) In this particular application, an additional "squeeze" ring is placed symmetrically at the longitudinal center of the cylinder. Squeeze voltages of various magnitudes are applied to this ring. Beyond the end-rings (longitudinally) and away from the plasma on each end is a short buffer zone of grounded cylinder at the end of which periodic boundary conditions $(\partial \phi / \partial z=0)$ are maintained to complete the boundary conditions for the computation region.

The azimuthal symmetry of the distribution of particles is broken by displacing particles a small amount from their equilibrium positions to seed an $m=1$ mode. This is done during an initial 500 time steps of 3 ns each in which the particles are incrementally displaced in opposite directions on each side of the median plane by $0.0002 \times r_{p}$ in each time step. By making the displacements in small increments over several bounce cycles of the particle motion, trapped particles experience a systematic displacement in a single direction from the axis (oppositely directed on the two sides of the median plane) while for untrapped particles the total displacement is essentially nulled.

Poisson's equation is solved by distributing density to the computational grid and using a three-dimensional multigrid algorithm to solve Poisson's equation. ${ }^{13}$ Particles are moved in the $(x, y)$ plane assuming $\mathbf{E} \times \mathbf{B}$ drift motion and using a predictor-corrector algorithm. In the longitudinal $z$ direction we use Newton's second law and a leap-frog algorithm. Densities are distributed to the grid, fields are computed from Poisson's equation, particles are moved in response to the fields, and new densities are computed to begin the cycle anew.

The Courant condition $\left(\omega_{p} \Delta t<1\right)$ is monitored to ensure that the code is numerically stable. The code was found to be very stable and once a somewhat optimized set of convergence parameters for the multigrid algorithm were chosen, the basic stepping code performed without further attention. For the test cases we describe in this paper, a 3 ns time step was typically used to follow about 5-10 cycles of the 
$m=1$ mode of interest. Over the course of a typical $75 \mu \mathrm{s}$ run, the average kinetic energy of the particles rises about $1 \%$ (the perturbation displaces the plasma from equilibrium and this may be related to the slight heating) and the angular momentum is conserved to within about $0.01 \%$. The conservation of angular momentum is checked by comparing the angular momentum at the end of the run with the angular momentum at the beginning of the run, but after the perturbation has been applied. Each simulation to determine a growth rate typically took several days of computer time.

The radius of the cylindrical trap used is $0.035 \mathrm{~m}$, with an overall length of the computing region of $0.40 \mathrm{~m}$. The confining rings are $0.03 \mathrm{~m}$ in width and begin $0.01 \mathrm{~m}$ in from either end of the computing region. The $0.05 \mathrm{~m}$ squeeze ring is centered symmetrically at the longitudinal middle of the cylinder. With a temperature of $1 \mathrm{eV}$, magnetic field of 0.04 $\mathrm{T}$, a voltage of $-100 \mathrm{~V}$ on the confining rings, and $-7.5 \mathrm{~V}$ on the squeeze ring, the simulated plasma has a length of $0.26 \mathrm{~m}$, a radius of $0.012 \mathrm{~m}$, and a central potential of approximately $-15 \mathrm{~V}$. The central density near the midpoint of either half of the plasma is $9.6 \times 10^{12} \mathrm{~m}^{-3}$. The total number of particles in the plasma is $6.7 \times 10^{8}$. The Debye length is of the order of $0.002 \mathrm{~m}$ and is comparable to the grid resolution in the longitudinal dimension and about twice the grid resolution in the perpendicular dimensions. The thermal velocity of the particles is $4.2 \times 10^{5} \mathrm{~m} / \mathrm{s}$. The gyroradius is of the order of $50 \mu \mathrm{m}$ or less for plasmas in this regime of parameter space and is ignored.

For this choice of parameters, the trapped-particle asymmetry mode frequency is $7.5 \times 10^{5} \mathrm{rad} / \mathrm{s}$, a typical bounce frequency is $5 \times 10^{6} \mathrm{rad} / \mathrm{s}$, a trapped particle rotation frequency at a radius of $0.01 \mathrm{~m}$ is $9.4 \times 10^{5} \mathrm{rad} / \mathrm{s}$, and a comparable untrapped particle rotation frequency is $1.1 \times 10^{6}$ $\mathrm{rad} / \mathrm{s}$. The value of the rotation frequency profile taken at the wall near the middle of one half of the plasma is $1.8 \times 10^{5}$ $\mathrm{rad} / \mathrm{s}$.

\section{RESULTS}

In Fig. 2 we show the results of computing the asymmetry mode frequencies as a function of squeeze voltage and two values of magnetic field. Since $\omega \propto B^{-1}$ and the simulations take a long time to run, we work at the low end of the field range covered by experiment.

In Fig. 3 we show the corresponding computed decay constants for the points in Fig. 2. Qualitatively, the figures correspond very well to the experimental results of Kabantsev et al. ${ }^{5,6}$ The decay constants decrease with increasing magnetic field as the more recent data show.

Our interest here is to shed light on the mechanism of the decay of the mode. For that purpose, we sought to identify two initial groups of particles. Trapped particles were identified as those particles whose orbits were confined to one side or the other of the median plane, while untrapped particles were those whose orbits were not limited to one side or the other. At the beginning of the evolution of the plasma and after the displacements that seed the mode, all particles that would encounter an insurmountable energy barrier if they moved along a magnetic field line to the median

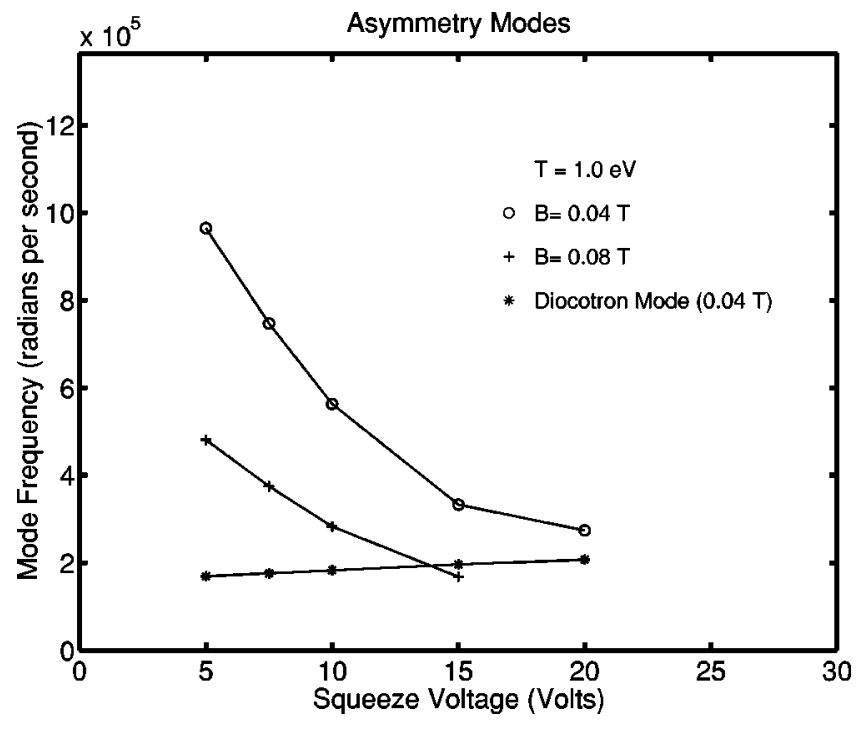

FIG. 2. Trapped-particle asymmetry mode frequencies as a function of squeeze voltage for two values of magnetic field. Asterisks mark corresponding $m=1$ diocotron frequencies taken from the cylinder wall values of the rotation frequency profiles. (For electrons, the squeeze potentials are negative.)

plane were marked as "trapped." Of course, any radial motion of the particles could cause it subsequently to become "untrapped" since the energy barrier is a function of radius.

In Fig. 4 we show the potential energy barrier (for electrons) as a function of radius (solid line). The curve is the potential energy taken at the midpoint of the plasma and terminates at the wall at a value determined by the squeeze voltage (in this case, $-7.5 \mathrm{~V}$ ). Also shown (dashed) is the potential energy profile taken near the midpoint of one half of the plasma (at about one-fourth of the plasma length.) In order for a particle to surmount the barrier, it must have a total energy that is greater than the solid curve. This difference must be made up by the kinetic energy of the particle. Near $R=0$ the difference between the dashed and solid

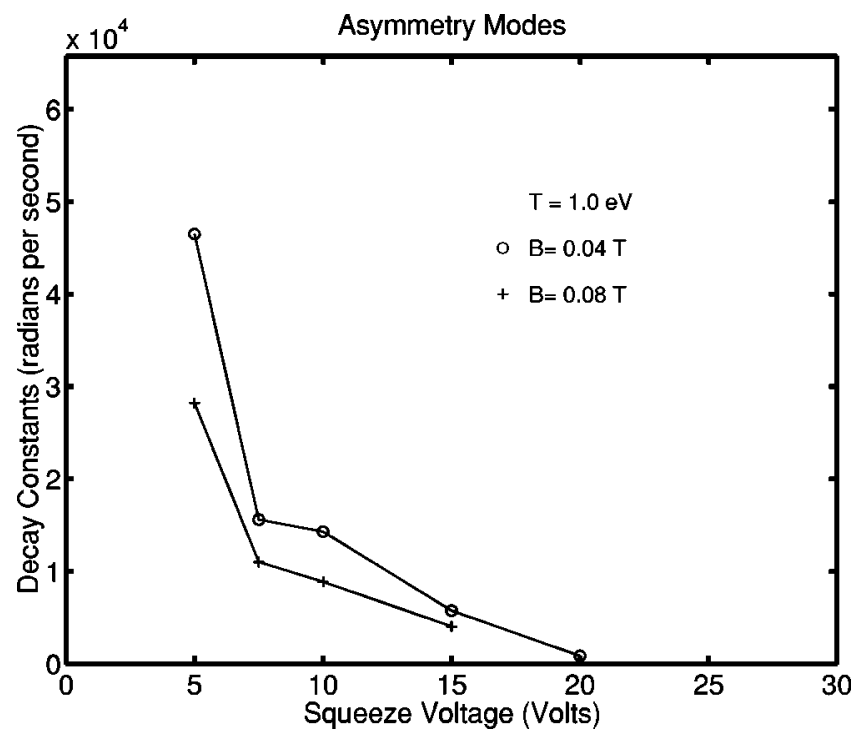

FIG. 3. Decay constants as a function of squeeze voltage for two values of magnetic field. (For electrons, the squeeze potentials are negative.) 


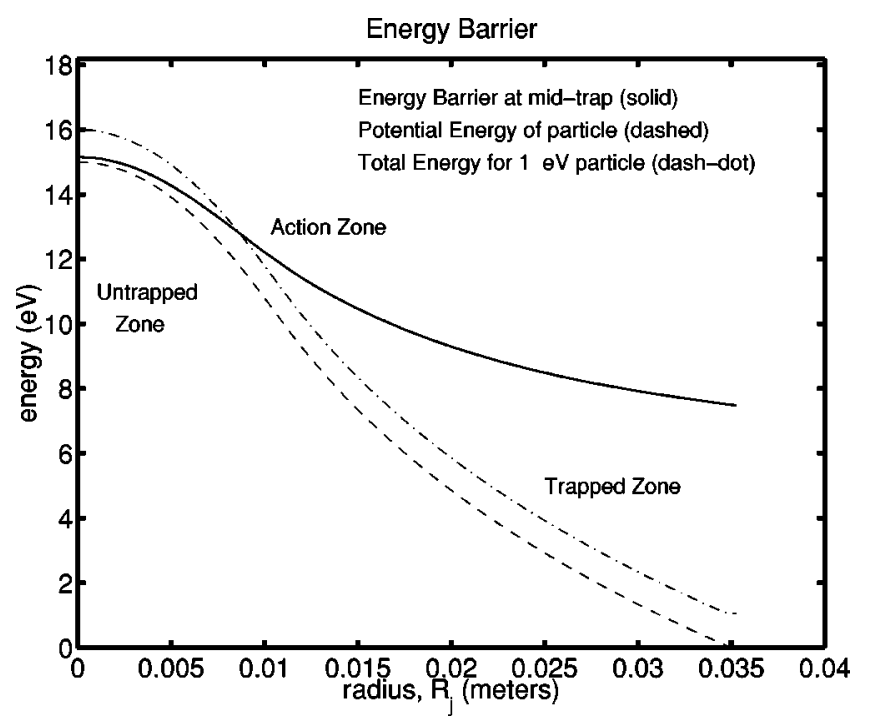

FIG. 4. The potential energy barrier seen by an electron at the middle of the trap (solid curve) falls to the energy determined by the squeeze voltage. For a particle located at the middle of either the left-hand or right-hand side of the plasma (at about a quarter of the total length of the plasma), the potential energy (dashed curve) falls to zero as a function of radius. For a particle to surmount the barrier, it must have sufficient kinetic energy added to its potential energy. The dashed-dotted line shows $1 \mathrm{eV}$ added to the electrical potential energy of the particle. The temperature of the plasma is $1 \mathrm{eV}$, the magnetic field is $0.04 \mathrm{~T}$, and the squeeze voltage is $-7.5 \mathrm{~V}$.

curves is so small that even a very small longitudinal velocity is sufficient to cross the barrier. This zone is labeled as the "untrapped zone." Particles in this zone are almost all untrapped and, for the most part, do not make transitions from trapped-to-untrapped or untrapped-to-trapped.

On the other hand, particles that find themselves at very large radius enter a "trapped" zone. For these it is unlikely that a particle will acquire enough longitudinal kinetic energy from longitudinal velocity fluctuations to cross the barrier. Particles deep enough in the "trapped zone," for the most part, do not make transitions between trapped and untrapped states.

The "action zone" is the fairly broad region in the middle where particles can relatively easily gain or lose sufficient longitudinal kinetic energy to make trapped-tountrapped or untrapped-to-trapped transitions. These two types of transitions have separate and distinct consequences for the radial transport of particles and decay of the mode. As we see in the following, these transitions are the essential "events" that cause particle transport and the decay of the mode. Slow fluctuations in particle velocity, primarily from diffusion in velocity space, make these essential events possible. However, the amount of kinetic energy needed by each particle depends strongly on its radial position, and, as it transports inward and outward, on time.

For each particle there is a "critical velocity" needed to surmount the barrier. This is a fairly sharp function of radial position and, as the mode decays, a function of time as the potential energy curves shift in response to the shifting plasma. Essentially, each particle has a "separatrix" in velocity space such that shifts back and forth across this critical velocity cause changes from trapped-to-untrapped or

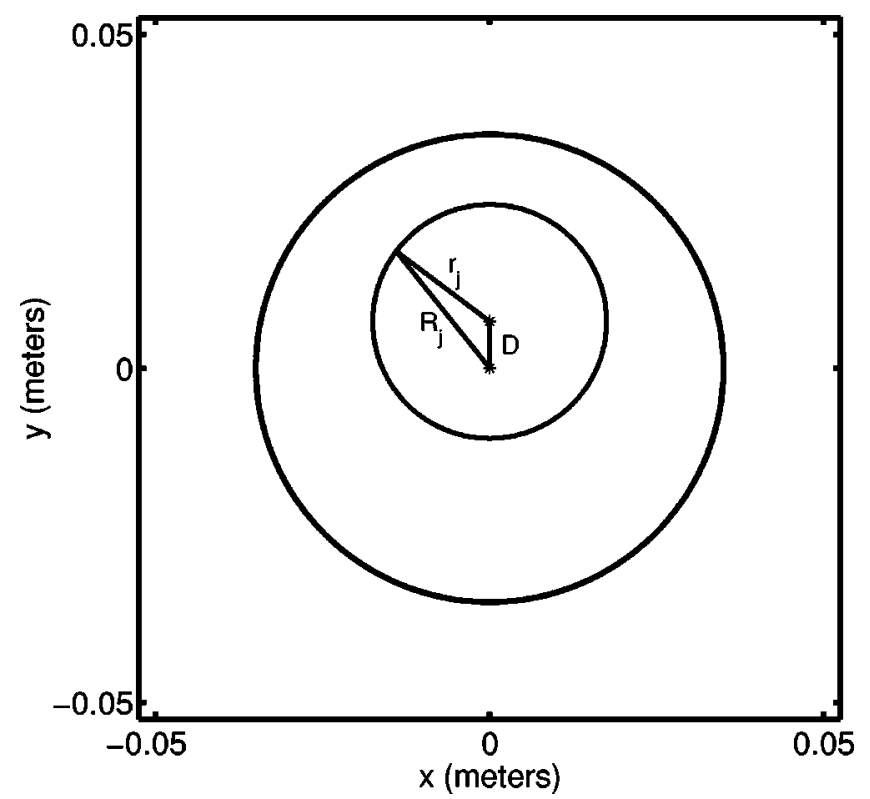

FIG. 5. The $m=1$ mode displaces the charge center of trapped particles relative to the symmetry center of the trap. Trapped particles revolve in circles of radius $r_{j}$ around the displaced charge center. The orbit of a trapped particle about the charge center creates a radial oscillatory motion whose amplitude is the mode amplitude, $D . R_{j}$ is the radius of a particle relative to the symmetry center. Untrapped particles revolve in circles around the symmetry center of the trap. Transitions from trapped to untrapped state or from untrapped to trapped state result in radial transport.

untrapped-to-trapped states. For a given particle in the action zone, fluctuations cause multiple passes over this separatrix during the evolution of the plasma.

As the plasma evolves, the particles initially marked as trapped were monitored periodically as a diagnostic to see if they had crossed over the median plane contrary to their classification. In the case where the squeeze voltage is -7.5 $\mathrm{V}$, about two-thirds of the particles were initially classified as "trapped." The simulations show that there is a steady streaming of so-called "trapped" particles across the median energy barrier as a function of time. There is no systematic dependence on magnetic field to this process (for fields ranging from 0.04 to $1000 \mathrm{~T}$ ) and only a weak dependence on temperature for temperatures ranging from 0.5 to $2.0 \mathrm{eV}$.

It is instructive to plot the radius of individual trapped and untrapped particles as a function of time. Figure 5 shows the displaced charge center of the trapped particles in one half of the trap relative to the symmetry center of the trap. Trapped particles revolve in nearly circular orbits around the displaced charge center, while untrapped particles revolve in nearly circular orbits around the symmetry center. For trapped particles this means that their orbits relative to the symmetry center oscillate between $r_{\max }$ and $r_{\min }$ with an amplitude equal to the mode amplitude. Figure 6 shows a plot of radius (relative to the symmetry center of the trap) versus time for a trapped particle and an untrapped particle taken from the simulations. The sinusoidal track belongs to a trapped particle, i.e., one that bounces back and forth through the entire evolution of the plasma between an end and the energy barrier at the median plane. Its motion is roughly circular around the displaced center of the $m=1$ mode and, 


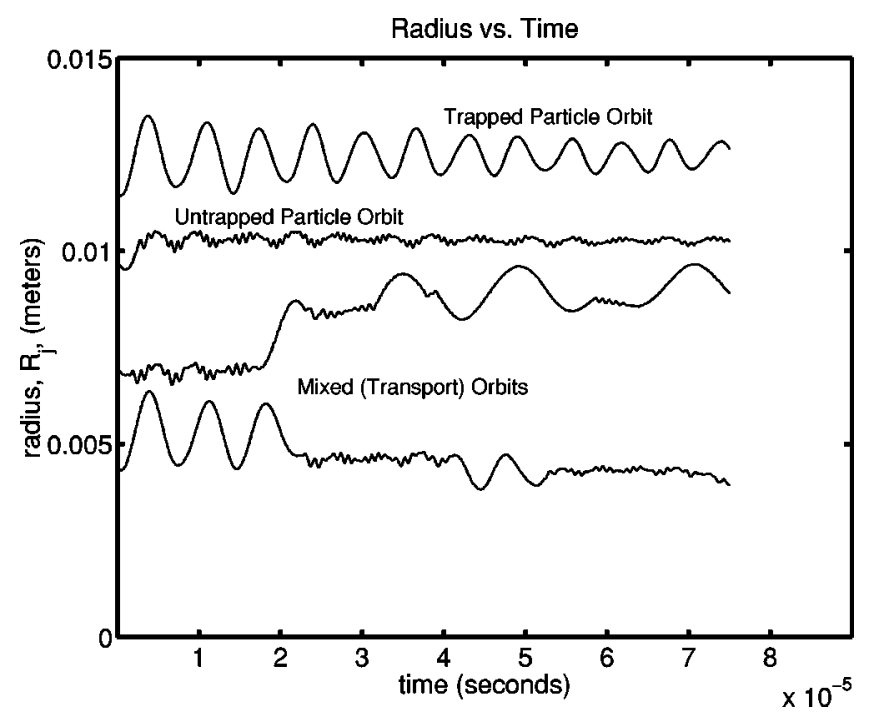

FIG. 6. Radius, $R_{j}$ (relative to the symmetry center of the trap) vs time for individual particles. The sinusoidal track at the top is the orbit of a trapped particle. The track at essentially constant radius is an untrapped particle. The remaining two tracks illustrate how transport occurs relative to the symmetry center when particles make transitions from trapped to untrapped status and back. These are the same particles shown in Fig. 7. The particle radii are repositioned for clarity from their true values near $r=0.01 \mathrm{~m}$.

hence, oscillates between a maximum and minimum radius relative to the symmetry center of the cylinder. The amplitude of the swing is just the amplitude of the mode itself and decreases with time as the mode amplitude decreases. On the other hand, the track of nearly constant radius belongs to an untrapped particle, i.e., one that bounces from end to end of the trap. Radial transport relative to the symmetry center of the trap occurs when a particle makes a transition from a trapped state to an untrapped state. The transport may be outward or inward depending on the phase of the sinusoidal motion when the particle makes a transition from trapped to untrapped state. (See Fig. 6.)

The canonical angular momentum of the system is given approximately by ${ }^{14}$

$$
P_{\theta} \approx \frac{e B}{2} \sum_{j=1}^{N} R_{j}^{2} \approx \frac{e B}{2} N\left(D^{2}+\frac{1}{N} \sum_{j=1}^{N} r_{j}^{2}\right),
$$

where $\mathbf{R}_{j}$ is the position of the $j$ th particle measured from the symmetry axis of the cylinder, $\mathbf{D}$ is the position of the $m$ $=1$ mode (charge) center, and $\mathbf{r}_{j}$ is the position of the particle relative to the mode center, i.e., $\mathbf{R}_{j}=\mathbf{D}+\mathbf{r}_{j}$.

Since the angular momentum is conserved in the simulations, the outward transport of any particles must be compensated by an inward transport of other particles relative to the symmetry center of the trap. However, the relative energy barrier that a particle must surmount to be or become untrapped is greater as the particle moves outward and lesser if the particle moves inward (finally approaching zero at $R$ $=0)$. Particles that move outward in radius $\left(R_{j}\right.$ increases $)$ eventually become trapped and are no longer able to acquire enough kinetic energy (through fluctuations) to cross the barrier. For these particles, no further transport is possible because the essential change from trapped-to-untrapped status

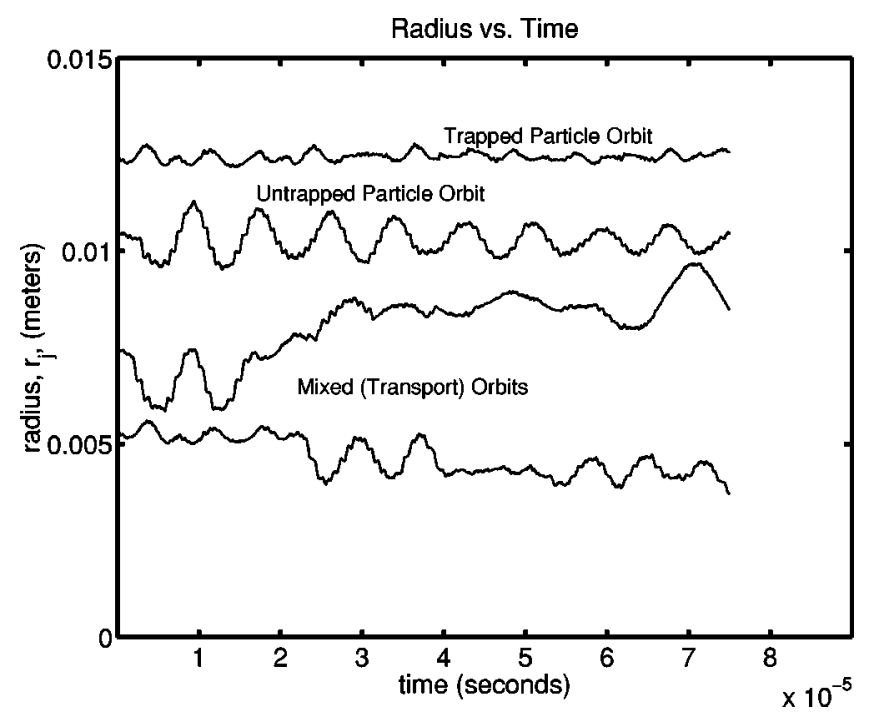

FIG. 7. Radius, $r_{j}$ (relative to mode center) vs time for individuals particles. The relatively flat track at the top is the orbit of a trapped particle. The track at essentially constant radius is an untrapped particle. The remaining two tracks illustrate how transport occurs relative to the mode center when particles make transitions from untrapped to trapped status and back. These are the same particles shown in Fig. 6. The particle radii are repositioned for clarity from their true values near $r=0.01 \mathrm{~m}$.

is no longer possible. For particles that move sufficiently toward the center, the barrier diminishes and they become essentially untrapped. For these particles, further transport also ceases because transitions again are no longer possible. At a radius of about $R=0.01 \mathrm{~m}$, the relative energy barrier to be surpassed by a particle is a few tenths of an eV. As $R$ increases, this relative barrier increases toward a limit determined by the squeeze voltage (several electron volts near the wall) and approaches zero as one approaches $R=0$. Small, relatively slow fluctuations of several tenths of electron volt (occurring over multiple bounce times and rotation periods) in parallel particle velocity provide the mechanism to allow a particle to cross the barrier, particularly in the vicinity of $R$ $=0.01 \mathrm{~m}$.

Since the mode amplitude $D$ decreases, the conservation of angular momentum also requires that the net effect must be for the particles to expand relative to the charge center, i.e., $\Sigma r_{j}^{2}$ must also increase. [See Eq. (1).] In Fig. 7 we show the radii of orbits of the same particles shown in Fig. 6, but plotted relative to the mode center. In this figure, a trapped particle has a relatively constant $r_{j}$ and an untrapped particle has an oscillatory $r_{j}$. Thus, for a transport event to occur, the particle must make an untrapped-to-trapped transition. This is just the opposite from the trapped-to-untrapped transition that creates a transport event relative to the symmetry center as measured by $R_{j}$.

If the kinetic and electrical potential energies of an individual particle are plotted (Fig. 8), the crossing of the energy barrier can be seen. (The energy history shown is for the third particle from the top in both Figs. 6 and 7.) There is a slow, fluctuating ebb and flow of the particle kinetic energy that does not display any obvious frequency dependence. When the particle is untrapped, there is superimposed on top of the slow fluctuations a cyclical modulating pattern that 


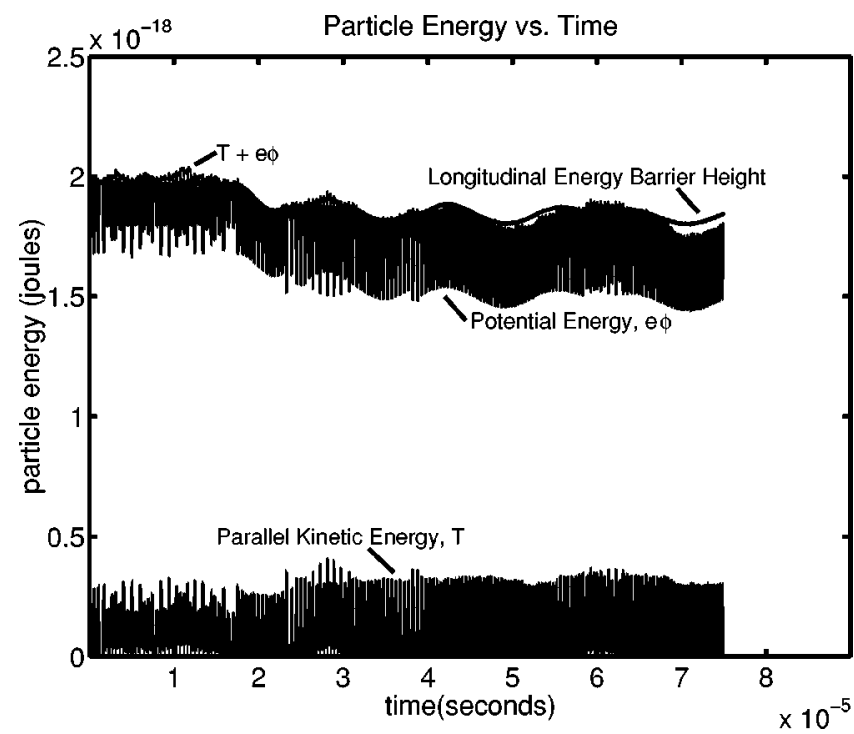

FIG. 8. The time dependence of the energy of a particle. The trace at the top of the graph is the midplane energy barrier height as viewed from the instantaneous position of the particle. The slightly noisy track that is sometimes below, sometimes above the barrier trace is the sum of parallel kinetic and electrical potential energies of the particle. As this trace crosses the barrier trace, the particle changes its trapped-untrapped state. Just below the total energy trace is the electrical potential energy history. Near the bottom of the graph is the parallel kinetic energy history of the particle. The traces are the energy history of the particle with the third orbit from the top in Fig. 6.

matches the frequency of the trapped-particle mode. It appears as a brief toothed pattern in the kinetic energy in Fig. 8 lasting during an untrapped episode from about 2.8 to 3.0 $\times 10^{-5}$ s. From observations of many other individual particle orbits we can say that if this particle were to remain untrapped for a longer period of time, this modulation would repeat itself. It comes about as the untrapped particle moves from the low electric potential energy side to the high potential energy side of the asymmetrical mode as it crosses the median plane. The frequency of the modulating pattern (which actually requires two repetitions of the toothed pattern in the figure to complete) decreases with radius and in all of the cases we have observed, is either $3 / 4 \omega_{m}, 4 / 4 \omega_{m}, 5 / 4 \omega_{m}$, or $6 / 4 \omega_{m}$. A particle crosses the median if it reaches the median plane with enough energy to surmount the energy barrier. Its radial position, the slow modulations of the particle's kinetic energy, and the toothed modulating pattern all combine to make this possible.

The radial swing amplitude of the orbit of a trapped particle depends on the amplitude of the $m=1$ displacement. The top trace in Fig. 6 shows that this amplitude decreases with time as the mode decays. The larger the mode displacement, the greater the swing from $r_{\min }$ to $r_{\max }$ and the greater the possible transport step if the particle surmounts the barrier. As the mode decays, the simulations show that the exponential decay constant gradually decreases with time, although the decrease over the length of the simulation runs is only of the order of $10 \%$.

Returning to Fig. 2, we observe that the mode frequency scales with increasing magnetic field as $B^{-1}$. However, Fig. 3 shows a weaker dependence for the decay constant with

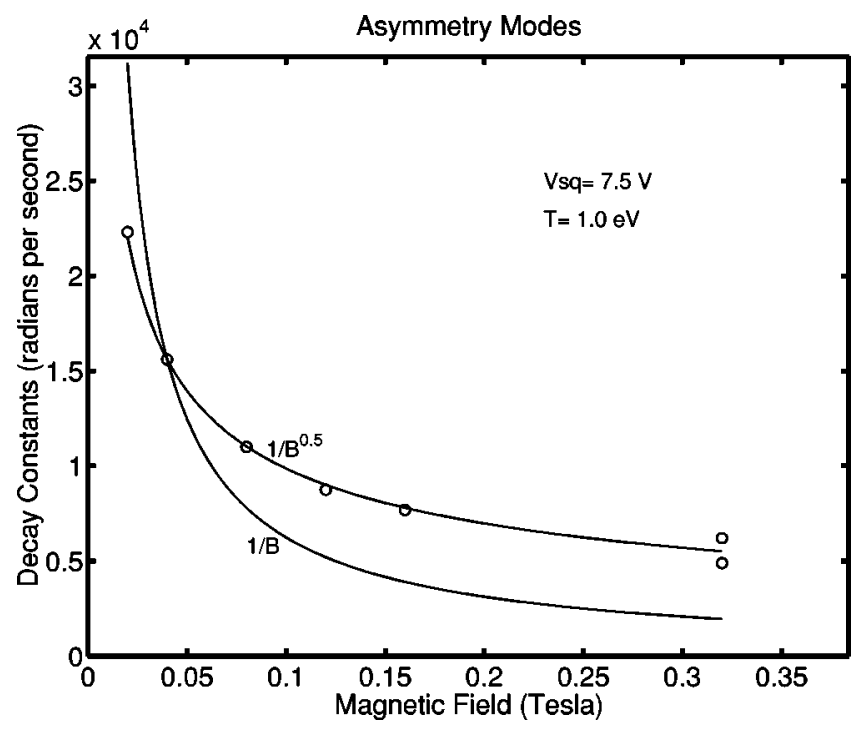

FIG. 9. Decay constants as a function of magnetic field for constant squeeze voltage $(7.5 \mathrm{~V})$ and temperature $(1 \mathrm{eV})$. The curves shown are for a $B^{-1}$ and $B^{-0.5}$ dependence normalized to the point at $0.04 \mathrm{~T}$ (our usual operating magnetic field).

magnetic field at fixed squeeze voltage. Overall, the scaling with magnetic field for the decay constant is probably consistent with a dependence that goes as $B^{-0.5}$ over the range of squeeze voltages used here. The average decrease factor when the field is doubled for the four squeeze voltages shown is 1.52 compared to $\sqrt{2}$ as it would be for a $B^{-0.5}$ scaling. The rigidity (ratio of bounce frequency to drift frequency) is about 10 (if one uses the mode frequency as a typical drift frequency) for the weaker magnetic field (0.04 $\mathrm{T})$ and twice that for $0.08 \mathrm{~T}$.

To further confirm the $B^{-0.5}$ behavior of the decay constants with magnetic field, we fix the squeeze voltage at $-7.5 \mathrm{~V}$, the temperature at $1 \mathrm{eV}$ and vary the magnetic field from 0.02 to $0.32 \mathrm{~T}$. The uppermost magnetic field on this range represents a practical limit for our code because of the inordinate computer time required to track the slow revolution of the plasma at high magnetic field. In Fig. 9 the dependence of the decay constant as a function of magnetic field is shown. For comparison, curves representing a $B^{-1}$ dependence and a $B^{-0.5}$ dependence are shown. Both curves are normalized at our "standard" magnetic field of $0.04 \mathrm{~T}$. This time, the fit to $B^{-0.5}$ out to $0.16 \mathrm{~T}$ is unmistakable. As noted earlier, the decay constants emerging from the simulations have a weak time dependence. To arrive at the numbers plotted in Fig. 9, we made an exponential fit to six different time intervals from zero to each of 50, 55, 60, 65, 70 and 75 $\mu \mathrm{s}$ and then took the average. This procedure also helped average out some jitter in the mode amplitude signal. For magnetic fields out to $0.16 \mathrm{~T}$, the variations with time were small (of the order of $10 \%$ ) so the effect was small. However, when the magnetic field was increased to $0.32 \mathrm{~T}$, we did not do this, since at $50 \mu$ s the plasma had not yet completed even a single revolution. Hence, for this stronger field we took the decay constant from two intervals, one extending to the end of the first revolution and the second extending to the end of the second. In this instance the decrease of the decay 


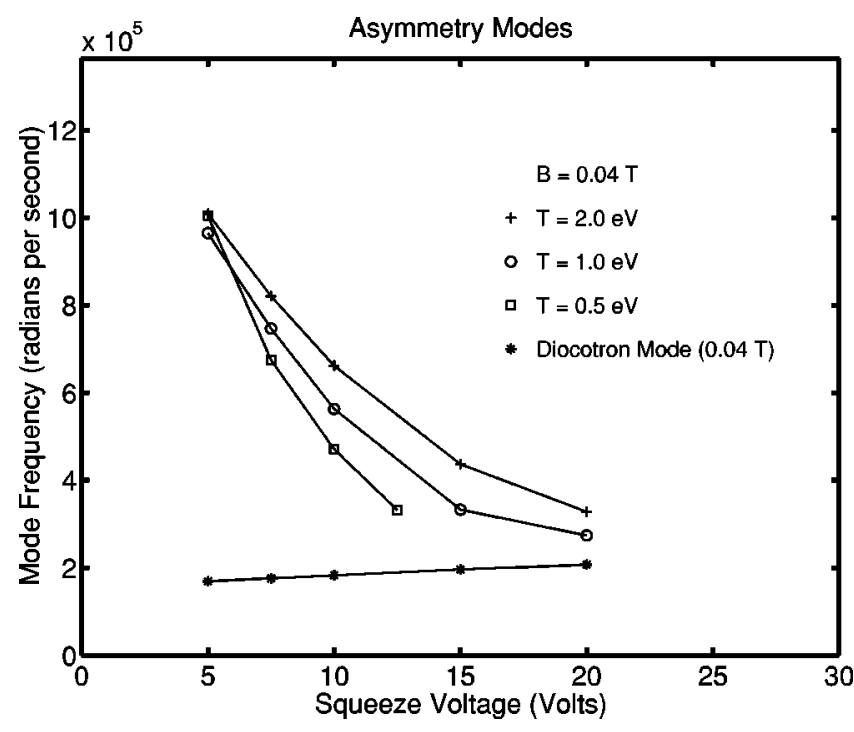

FIG. 10. The mode frequency as a function of squeeze voltage for different temperatures. Except for the smallest squeeze voltage, the mode frequencies increase with temperature in a systematic way. (For electrons, the squeeze potentials are negative.)

constant was about $20 \%$ according to this protocol. Both points are shown in Fig. 9 at $0.32 \mathrm{~T}$.

Figure 10 shows the dependence of the mode frequency with temperature in the simulations over a range $0.5-2.0 \mathrm{eV}$. Except at the very lowest squeeze, the frequency shows a clear systematic enhancement with increasing temperature for given squeeze voltage. On the other hand (see Fig. 11), the dependence of the decay constant with temperature in the simulations is much less pronounced and exhibits a "crossover" in behavior as a function of squeeze voltage.

The slow variations in an individual particle's parallel velocity that can be seen in the parallel kinetic energy trace in Fig. 8 come about because of the random walk of the particle in one-dimensional longitudinal velocity space, i.e., from diffusion. Crudely, the longitudinal $\Delta v$ for such a random walk would be proportional to the square root of the longitudinal collision frequency $\sqrt{\nu}$ and square root of the density $n$, so that $\Delta v$ would depend on the charge, mass, and density of the particles as $n^{0.5} q^{2} / m$. However, a PIC code moves "super particles" that each represent some number $N_{e}$ of real electrons. The super particles have a charge $Q$ $=N_{e} q$, mass $M=N_{e} m$, and a density $N=n / N_{e}$. The collisions of the simulation therefore exaggerate velocity transfers by a factor $N_{e}^{0.5}$. Thus, effects in PIC simulations that might depend on the details of the collisionality cannot be expected necessarily to be directly comparable with experiment. Such is the case here.

\section{CONCLUSIONS}

The decay mechanism for trapped-particle asymmetry modes is seen to be an orbital dynamics effect driven by longitudinal collisions. Trapped particles make nearly circular orbits around the displaced charge center of the mode. These orbits carry the particles cyclically between minimum and maximum radii relative to the symmetry axis of the trap.

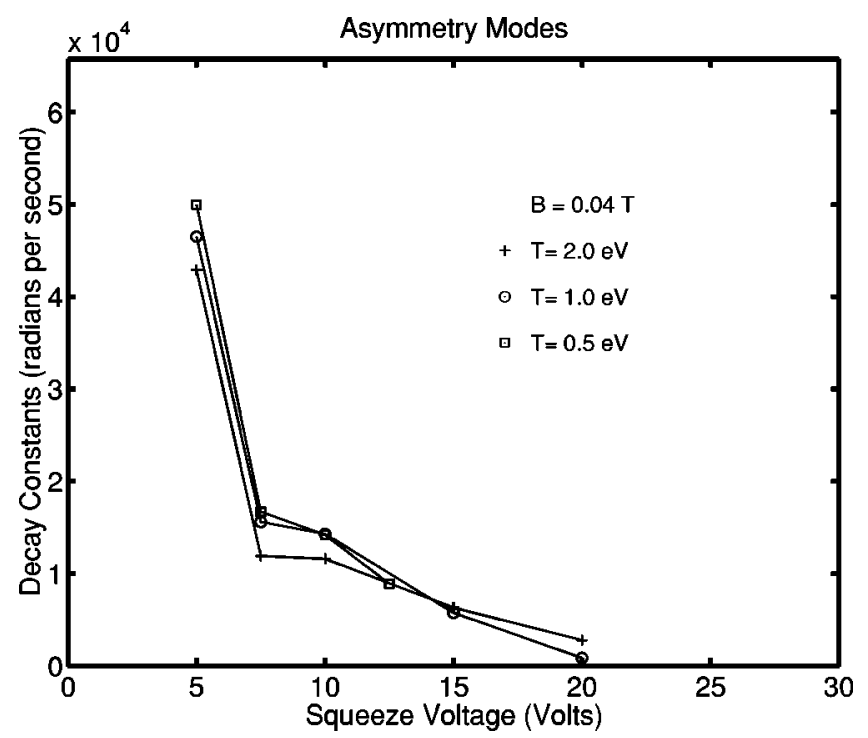

FIG. 11. The decay constants as a function of squeeze voltage for different temperatures. For this limited range of temperatures, the dependence on temperature is small and somewhat ambiguous in scaling behavior for different squeeze voltages. (For electrons, the squeeze potentials are negative.)

The amplitude of the radial swings is just the amplitude of the mode. Random walks of the particle in velocity space can give a trapped particle sufficient energy to change from trapped to untrapped state and back. The transport and accompanying decay of the trapped-particle asymmetry mode could be treated theoretically as a collision process, even though the mechanism is not a result of immediate individual Coulomb collisions. The transitions from trapped to untrapped particle states play the role of collisions and the (time dependent) amplitude of the mode gives the scale of the step size.

Transitions from trapped-to-untrapped states give rise to transport relative to the symmetry axis of the trap, but transitions from untrapped-to-trapped states give rise to transport relative to the mode center. Conservation of angular momentum in the system requires that the mode amplitude decrease if the plasma expands relative to the mode center.

Slow modulations of the kinetic energy of the particles and velocity "kicks" as the particles move from the high potential energy end to the low potential energy end of the asymmetric mode make it possible for particles to make repeated transitions from trapped to untrapped and back.

\section{ACKNOWLEDGMENTS}

We gratefully acknowledge the use of facilities of the Brigham Young University Supercomputing Center. We also acknowledge helpful discussions and encouragement from R. L. Spencer, C. F. Driscoll, T. J. Hilsabeck, and A. A. Kabantsev.

${ }^{1}$ R. H. Levy, Phys. Fluids 8, 1288 (1965); 11, 920 (1968).

${ }^{2}$ R. J. Briggs, J. D. Daugherty, and R. H. Levy, Phys. Fluids 13, 421 (1970).

${ }^{3}$ R. C. Davidson, Theory of Nonneutral Plasmas (Benjamin, Reading, MA, 1974).

${ }^{4}$ K. S. Fine, Ph.D. thesis, University of California at San Diego, 1989.

${ }^{5}$ A. A. Kabantsev, C. F. Driscoll, T. J. Hilsabeck, T. M. O'Neil, and J. H. 
Yu, in Non-Neutral Plasmas IV, edited by F. Anderegg, L. Schweikhard, and C. F. Driscoll (American Institute of Physics, New York, 2002), pp. 277-286.

${ }^{6}$ A. A. Kabantsev, C. F. Driscoll, T. J. Hilsabeck, T. M. O'Neil, and J. H. Yu, Phys. Rev. Lett. 87, 225002 (2001).

${ }^{7}$ A. A. Kabantsev and C. F. Driscoll, Bull. Am. Phys. Soc. 47, 125 (2002).

${ }^{8}$ A. A. Kabantsev and C. F. Driscoll, Phys. Rev. Lett. 89, 245001 (2002).

${ }^{9}$ T. J. Hilsabeck and T. M. O'Neil, Bull. Am. Phys. Soc. 47, 125 (2002).
${ }^{10}$ G. W. Mason and R. L. Spencer, Bull. Am. Phys. Soc. 47, 125 (2002).

${ }^{11}$ G. W. Mason and R. L. Spencer, Phys. Plasmas 9, 3217 (2002).

${ }^{12}$ R. L. Spencer, S. N. Rasband, and R. R. Vanfleet, Phys. Fluids B 5, 1738 (1993).

${ }^{13}$ W. H. Press, S. A. Teukolsky, W. T. Vetterling, and B. P. Flannery, $N u-$ merical Recipes in Fortran, 2nd ed. (Cambridge University Press, New York, 1992), Chap. 10.

${ }^{14}$ S. M. Crooks and T. M. O’Neil, Phys. Plasmas 2, 355 (1995). 